\title{
Treatment of Axillary Hyperhidrosis/Bromidrosis Using VASER Ultrasound
}

\author{
Julio Garcia
}

Published online: 31 December 2009

(c) Springer Science+Business Media, LLC and International Society of Aesthetic Plastic Surgery 2009

I would like to commend Dr. Commons and Dr. Lim (Treatment of Axillary Hyperhidrosis/Bromidrosis using VASER Ultrasound, Aesthetic Plastic Surgery) on their analytical approach to a problem that obviously affects a large portion of the population. Their well-documented protocols and patient assessment forms yield objective criteria which make it easy to understand and attempt to ascertain efficacy of this problem. Their clear method of describing how the procedure is performed with actual treatment energies and times is also to be commended. These small little details are crucial for those considering their own trials in as safe of a protocol as possible and are usually missing in many presentations by others when using energy sources, whether ultrasound or lasers. Being well-versed with internal ultrasonic disruption of fat cells, both VASER and the other technologies, for over 12 years I too am always looking to new potential applications for this technology. With this being said, I would like to pose some questions that might be able to be addressed by Dr. Commons and Dr. Lim in a follow up to this publication.

Understanding that fibrous areas with dense septae require longer treatment times with the ultrasonic modality, I certainly understand that longer times would be required in this location due to its dense adherence, but I would be curious to hear the authors' comments on the phases and what each phase accomplished. I would agree that discontinuous disruption for proper eventual cannula application is crucial, so would they say that the first two phases allowed the truly effective phase of the focused energy to be applied to the sweat glands during the back stroke curettage phase? One would think that the angled direction on the back stroke

J. Garcia $(\bowtie)$

6020 S. Rainbow, Las Vegas, NV 89118, USA

e-mail: jgarciamd@aol.com would be where the focused energy could have caused the damage to the sweat glands and not as much during the parallel stroke phase. I would also like to hear their comment on how much they feel the location being so close to lymphatics aided in the low seroma rates since we have found that longer treatment times occasionally lead to seromas in dense areas. The etiology of the damage would be nice to understand by pathology examination. We can theorize that some element of the fatty component in the sweat or oil glands was damaged by the energy since we have all come to understand that collagen and blood vessels are typically preserved during the use of this technology, but objective evidence in the future would be helpful. Was there ever any consideration to a pre- and post-treatment punch biopsy to evaluate the change in dermal and subdermal architecture to elucidate the effect on the glands by the focused energy? Although not commonly available yet, I would like to hear their comments on the potential for external focused fat dissolution technology and how it could be applied to this same problem with a lesser invasive procedure.

I think that comparing their pilot study in terms of costs to other modalities' projected costs over longer periods of time is a little premature. Perhaps once those treated patients return at 1 year and fill out the same assessments forms that information could be better assessed for comparable cost. Until such time, I would avoid the hyperbole, especially in the utilization of a technology that would be off-label and therefore not able to be marketed legally for that purpose.

Overall, I found Dr. Common's and Dr. Lim's paper exciting, well-documented and well thought out, with honest evaluation and well-described protocols. I anxiously await their follow-up to this initial paper as I think they have found an exciting treatment option for a problem that most of us have been frustrated with for many years. 